\title{
MICROBUNDLES AND THOM CLASSES
}

\author{
BY PER HOLM ${ }^{1}$ \\ Communicated by S. Smale, December 17, 1965
}

In this note we introduce Thom classes of microbundles. We determine the Thom class of the Whitney sum as the cup product of Thom classes and state two applications; one to Gysin sequences of Whitney sums and one to the Atiyah-Bott-Shapiro duality theorem for Thom spaces (cf. Atiyah [2]). Thus our main result states that for mictobundles $\mu_{1}, \mu_{2}$ over a compact (topological) manifold $X$, if $\tau(X) \oplus \mu_{1}$ $\oplus \mu_{2}$ is $J$-trivial, then $\mu_{1}$ and $\mu_{2}$ have $S$-dual Thom spaces. Actually our result is more general since it treats the relative case, i.e. with relative Thom spaces. This makes us able to handle manifolds with boundaries (by passing to the double) among other things. Thus proposition (3.2) in Atiyah [2] has an extended version which just appears as another special case of our duality theorem.

The approach given here to the $S$-duality theorem shows very clearly that $S$-duality of Thom spaces is simply Alexander-Spanier duality of compact pairs in the base manifold lifted by Thom isomorphisms. Our approach does not make use of imbeddings of manifolds, and we think it is conceptually easier than Atiyah's method, although there are some technical difficulties due to the fact that we work in a more general setting.

Throughout this paper all base spaces of bundles and microbundles are assumed paracompact unless otherwise stated. Manifolds are manifolds without boundary. For notations and concepts see [4].

Generalizations and details will appear elsewhere.

Throughout this paper $R$ denotes a fixed principal ideal domain. By a local system on a space $X$ we understand a local system of $R$-modules on $X$, i.e. a (contravariant) functor from the fundamental groupoid of $X$ to the category of $R$-modules. If

$$
\mu: X \stackrel{s}{\rightarrow} E \stackrel{p}{\rightarrow} X
$$

is an $R^{a}$-microbundle with total space $E$, write $E^{0}=E-s X$. Then there are local systems $\mathcal{O}=\mathcal{O}(\mu), \mathcal{O}^{*}=\mathcal{O}^{*}(\mu)$ on $X$ corresponding under pull-backs and depending only on the equivalence class of $\mu$, such that for $x \in X, \mathcal{O}_{x}=H^{q}\left(E\left|x, E^{0}\right| x\right), \mathcal{O}_{x}^{*}=H_{q}\left(E\left|x, E^{0}\right| x\right)$. (Coefficients in homology or cohomology are taken in $R$ if not indicated.)

1 This work was done in Berkeley, California, in spring 1965 while the author was supported from NAVF (Norway) and NSF contract OP-4035. 
These are the orientation systems of $\mu$. They are constant over each trivializing subset of $X$ but in general not constant over $X$.

A Thom class for the microbundle $\mu$ is a cohomology class $U \in H^{q}\left(E, E^{0} ; p^{*} \mathcal{O}^{*}\right)$ such that the restriction to $H^{q}\left(E\left|x, E^{0}\right| x ; \mathcal{O}_{x}^{*}\right)$ $\approx \operatorname{Hom}\left(H_{q}\left(E\left|x, E^{0}\right| x\right), H_{q}\left(E\left|x, E^{0}\right| x\right)\right)$ maps $U$ to the generator corresponding to the identity automorphism. It is not difficult to show the following

(1) Theorem. Any microbundle admits a unique Thom class.

Clearly Thom classes correspond under equivalences and pullbacks.

Let

$$
\mu_{1}: X \stackrel{s_{1}}{\rightarrow} E_{1} \stackrel{p_{1}}{\rightarrow} X, \quad \mu_{2}: X \stackrel{s_{2}}{\rightarrow} E_{2} \stackrel{p_{2}}{\rightarrow} X
$$

be two microbundles over $X$ and let

$$
\mu: X \stackrel{s}{\rightarrow} E \stackrel{p}{\rightarrow} X
$$

be their Whitney sum. Let $\pi_{1}: E \rightarrow E_{1}$ and $\pi_{2}: E \rightarrow E_{2}$ be the canonical projections and $\sigma_{1}: E_{1} \rightarrow E, \sigma_{2}: E_{2} \rightarrow E$ their corresponding sections. The projections $\pi_{1}, \pi_{2}$ define maps of pairs

$$
\bar{\pi}_{1}:\left(E, E-\sigma_{2} E_{2}\right) \rightarrow\left(E_{1}, E_{1}^{0}\right), \quad \bar{\pi}_{2}:\left(E, E-\sigma_{1} E_{1}\right) \rightarrow\left(E_{2}, E_{2}^{0}\right)
$$

which induce isomorphisms in homology. Given a pairing of local systems on $X \psi: S_{1} \otimes S_{2} \rightarrow S$, there is a natural pairing

$$
H^{*}\left(E, E_{1}^{0} ; p_{1}^{*} S_{1}\right) \otimes H^{*}\left(E_{2}, E_{2}^{0} ; p_{2}^{*} S_{2}\right) \rightarrow H^{*}\left(E, E^{0} ; p^{*} s\right)
$$

defined to be the composite

$$
\begin{gathered}
H^{*}\left(E_{1}, E_{1}^{0} ; p_{1}^{*} s_{1}\right) \otimes H^{*}\left(E_{2}, E_{2}^{0} ; p_{2}^{*} S_{2}\right) \\
\bar{\pi}_{1}^{*} \otimes \bar{\pi}_{2}^{*} \downarrow \\
H^{*}\left(E, E-\sigma_{2} E_{2} ; \bar{\pi}_{1}^{*} p_{1}^{*} S_{1}\right) \otimes H^{*}\left(E, E-\sigma_{1} E_{1} ; \bar{\pi}_{2}^{*} p_{2}{ }^{*} s_{2}\right) \\
U \downarrow \\
H^{*}\left(E, E^{0} ; \bar{\pi}_{1}^{*} p_{1}^{*} s_{1} \otimes \bar{\pi}_{1}^{*} p_{2}^{*} S_{2}\right) \\
\tau_{*} \downarrow \\
H^{*}\left(E, E^{0} ; p^{*} s\right) .
\end{gathered}
$$

The map $\cup$ is the ordinary cup product, and $\tau_{*}$ is the homomorphism induced from the composite 


$$
\tau: \bar{\pi}_{1}^{*} p_{1}^{*} S_{1} \otimes \bar{\pi}_{2}^{*} p_{2}^{*} \delta_{2}=p^{*} S_{1} \otimes p^{*} S_{2} \approx p^{*}\left(S_{1} \otimes S_{2}\right) \stackrel{p^{*} \psi}{\longrightarrow} p^{*} S .
$$

This pairing is invariant under equivalence of microbundles, but in general dependent on the germs of $p_{i}$ and $s_{i}, i=1,2$. The image of an element $u \times v$ will (by a slight abuse of notation) be denoted $\pi_{1}^{*}(u)$ $\cup \tilde{\pi}_{2}^{*}(v)$. It follows that there is always a pairing induced from the Künneth formula pairing $\Theta_{1}^{*} \otimes \mathcal{O}_{1}^{*} \rightarrow \mathcal{O}^{*}$. The following is true

(2) THEOREM. If $\mu, \mu_{1}, \mu_{2}$ are microbundles with Thom classes $U, U_{1}$, $U_{2}$ and $\mu=\mu_{1} \oplus \mu_{2}$, then $U=\bar{\pi}_{1}^{*}\left(U_{1}\right) \cup \bar{\pi}_{2}^{*}(U)$.

Thus, if $\bar{\Delta}: \mu_{1} \oplus \mu_{2} \rightarrow \mu_{1} \times \mu_{2}$ is the natural bundle map induced from the diagonal map $\Delta$ on the base, it follows that $\bar{\Delta}^{*}\left(U_{1} \times U_{2}\right)=\pi_{1}^{*}\left(U_{1}\right)$ $\cup_{\pi_{2}^{*}}^{*}\left(U_{2}\right)$.

By a spectral sequence argument (or by elementary piecingtogether techniques using a Mayer-Vietoris exact sequence) we can show the existence of a relative Thom isomorphism:

(3) THEOREM. Let

$$
\mu: X \stackrel{s}{\rightarrow} E \stackrel{p}{\rightarrow} X
$$

be an $R^{a}$-microbundle, $A$ a subset of $X$ such that $\left\{E^{0}, E \mid A\right\}$ is excisive and $S$ a local system on $X$. Then there are natural isomorphisms of graded modules of degrees $-q$ and $q$ respectively

$$
\begin{aligned}
& \phi: H\left(E, E^{0} \cup E \mid A ; p^{*} \varsigma\right) \approx H\left(X, A ; 0^{*} \otimes \varsigma\right),
\end{aligned}
$$

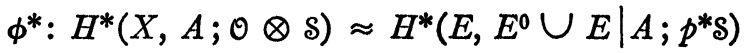

defined by

$$
\phi(z)=p_{*}\left(U_{\mu} \cap z\right), \quad \phi^{*}(v)=p^{*}(v) \cup U_{\mu} .
$$

Note that $\phi$ and $\phi^{*}$ are invariant under equivalence of microbundles.

The Thom class of $\mu$ when restricted to $X$ gives rise to an element $\Omega_{\mu} \in H^{q}\left(X ; \mathcal{O}^{*}\right)$ called the characteristic class of $\mu$. As an application of (2) we give the following relations between the Gysin sequences of a Whitney sum and those of the components. (For simplicity of notations we consider the bundle case only.)

(4) Theorem. Let $\xi_{i}, i=1,2$ be $R^{a_{i} \text {-bundles over a space } X \text { and }}$ $\xi=\xi_{1} \oplus \xi_{2}$ their Whitney sum

$$
\begin{aligned}
& \xi_{i}: X \stackrel{s_{i}}{\rightarrow} E_{i} \stackrel{p_{i}}{\rightarrow} X, \quad i=1,2, \\
& \xi: X \stackrel{s}{\rightarrow} E \stackrel{p}{\rightarrow} X .
\end{aligned}
$$


The canonical injections $\sigma_{i}: E_{i} \rightarrow E$ induce maps between the Gysin sequences of $\xi_{i}$ and $\xi$ :

$$
\begin{aligned}
& \cdots \rightarrow H_{n}\left(E_{i}^{0}, E_{i}^{0} \mid A ; \quad p_{i}^{0 *} \varsigma\right) \rightarrow H_{n}(X, A ; \delta) \rightarrow H_{n-q_{i}}\left(X, A ; \Theta_{i}^{*} \otimes \delta\right) \rightarrow \cdots \\
& \sigma_{i *}^{0} \downarrow \quad \| i d . \quad \psi_{i *} \downarrow \\
& \cdots \rightarrow H_{n}\left(E^{0}, E^{0} \mid A ; p^{0 *}\right) \rightarrow H_{n}(X, A ; s) \rightarrow H_{n-q}\left(X, A ; \mathcal{O}^{*} \otimes s\right) \rightarrow \cdots
\end{aligned}
$$

where the maps $\psi_{i *}$ are given by

$$
\psi_{1 *}(z)=(-1)^{q_{1} q_{2}} \Omega_{2} \cap z, \quad \psi_{2 *}(z)=\Omega_{1} \cap z .
$$

Similarly, in the diagrams of the Gysin cohomology sequences the corresponding maps $\psi_{i}^{*}$ are given by

$$
\psi_{1}^{*}(v)=(-1)^{q_{1} q_{2}} v \cup \Omega_{2}, \quad \psi_{2}^{*}(v)=v \cup \Omega_{2} .
$$

The verification is easy except for determining the $\psi_{i *}$ and $\psi_{i}^{*}$ which is done directly by means of Theorem 2 . Theorem 4 has been found by Chern and Lashof (unpublished) in the case of orientable orthogonal bundles over a compact manifold.

For any integer $q=0$ let $R^{a} C S^{q}$ be an imbedding of $R^{q}$ into its one-point compactification, fixed throughout this paper, and let $\infty$ denote the complementary point of $R^{q}$ in $S^{q}$. Moreover, let $G\left(R^{q}, 0\right)$, respectively $G\left(S^{q}, 0, \infty\right)$, denote the group of all homeomorphisms of $\boldsymbol{R}^{q}$, respectively $S^{q}$, which leave the origin 0 , respectively 0 and $\infty$, fixed. Any homeomorphism of $\left(R^{q}, 0\right)$ extends to a homeomorphism of $\left(S^{q}, 0, \infty\right)$. Therefore the imbedding $R^{a} \subset S^{q}$ induces an imbedding $G\left(R^{q}, 0\right) \subset G\left(S^{q}, 0, \infty\right)$ of topological groups. It follows that any $\boldsymbol{R}^{\boldsymbol{a}}$-bundle $\xi$ has a functorially associated $S^{q}$-bundle $\xi_{\infty}$ (over the same base) with two sections $s_{0}, s_{\infty}$, and that $\xi$ is naturally imbedded in $\xi_{\infty}$ with zero-section corresponding to $s_{0}$ and total space $E$ corresponding to $E_{\infty}-\mathrm{im} s_{\infty}$.

We make a remark about Thom spaces. If

$$
\xi: X \stackrel{s}{\rightarrow} E \stackrel{p}{\rightarrow} X
$$

is an $R^{q}$-bundle with associated $S^{q}$-bundle

$$
\xi_{\infty}: X \stackrel{s_{\infty}}{\rightarrow} E_{\infty} \stackrel{p_{\infty}}{\rightarrow} X,
$$

and $(A, B)$ is a pair of subsets of $X$, the Thom space $T_{\xi}(A, B)$ is defined to be the pointed space

$$
T_{\xi}(A, B)=p_{\infty}^{-1} A /\left(s_{\infty} A \cup p_{\infty}^{-1} B\right) .
$$


By the representation theorem in [4] this definition carries over to microbundles (cf. last part of [4]).

We next show that $T_{\xi}(A, B)$ reflects the homology properties of the bundle pair $(\xi|A, \xi| B)$, at least when $(A, B)$ is nicely imbedded in $X$. A radial neighbourhood of a closed set $B$ in $X$ is an open set $U \subset B$ such that $\bar{U}$ is homeomorphic to the mapping cylinder of a map $\dot{U} \rightarrow B$ ( $\dot{U}=$ boundary of $U$ ) sending $\dot{U}$ onto $\dot{B}$, by a homeomorphism which is the identity on $\dot{U} \cup B$. We will say that $B$ is radially imbedded or $r$-imbedded in $X$. Notice that $X$ and $\phi$ is always $r$-imbedded in $X$. A radial neighbourhood of a closed pair $(A, B)$ in $X(B \subset A)$ is an open pair $(U, V)$ with $U$, resp. $V$, a radial neighbourhood of $A$, resp. $B$. If $(A, B)$ is $r$-imbedded in $X$ with a radial neighbourhood $(U, V)$, then again $(X-V, X-U)$ is $r$-imbedded in $X$, and $H(U, V) \approx H(A, B), H(X-V, X-U) \approx H(X-B, X-A)$. Moreover, there is induced an $r$-imbedding of $\left(E_{\infty}\left|A, E_{\infty}\right| B\right)$ in $E_{\infty}$ with $r$-neighbourhood $\left(E_{\infty}\left|U, E_{\infty}\right| V\right)$, compatible with the $r$-structure of $s_{0}(A, B)$ and $s_{\infty}(A, B)$ in im $s_{0}$ and im $s_{\infty}$. From this we get the following:

(5) Lemma. Let

$$
\xi: X \stackrel{s}{\rightarrow} E \stackrel{p}{\rightarrow} X
$$

be an $R^{a-b u n d l e ~ a n d ~}(A, B)$ an $r$-imbedded closed pair in $X$. Then there is an isomorphism

$$
\widetilde{H}\left(T_{\xi}(A, B)\right) \approx H\left(E\left|A, E^{0}\right| A \cup E \mid B\right)
$$

natural with respect to fibrewise proper bundle maps $\xi\left|(A, B) \rightarrow \xi^{\prime}\right|(A, B)$.

By the microbundle representation theorem and a theorem of Hirsch and Mazur [3] (suitably generalized) the notion of $J$-equivalence can be defined for microbundles as well as for (orthogonal) vector bundles, and one shows that if $\mu, \mu^{\prime}$ are $J$-equivalent microbundles over $X$ then for any pair $(A, B)$ in $X, T_{\xi}(A, B)$ and $T_{\xi^{\prime}}(A, B)$ are of the same $S$-type.

We turn to the announced duality theorem. Let $X$ be a $q$-manifold and let $\mu_{1}, \mu_{2}$ be microbundles over $X$ such that $\mu=\tau \oplus \mu_{1} \oplus \mu_{2}$ is $J$-trivial. If $(A, B)$ is a compact pair $r$-imbedded in $X$ with $r$-neighbourhood $(U, V)$, we establish a map of pointed spaces

$$
T_{\mu_{1}}(A, B) \wedge T_{\mu_{2}}(X-V, X-U) \rightarrow\left(S^{Q}, \infty\right)
$$

where $Q=q+q_{1}+q_{2}$. To show that this is an $S$-duality map we should show that slanting with the spherical class in $\widetilde{H}^{Q}\left(T_{\mu_{1}}(A, B)\right.$ $\left.\wedge T_{\mu_{2}}(X-V, X-U)\right)$ defines an isomorphism

$$
\tilde{H}_{n}\left(T_{\mu_{2}}(X-V, X-U)\right) \approx \tilde{H}^{Q-n}\left(T_{\mu_{1}}(A, B)\right) .
$$


Instead we may look at the corresponding map, say $\gamma(A, B)$, under the isomorphisms of Lemma 5. Then the spherical class corresponds to the Thom class of $\mu$ properly restricted, and the result we are heading for follows from

(6) TheOREM. The map $\gamma=\gamma(A, B)$ is an isomorphism. More precisely we have the commutative diagram

$$
\begin{aligned}
& H_{n}\left(E_{2}\left|(X-B), E_{2}^{0}\right|(X-B) \cup E_{2} \mid(X-A)\right) \\
& \stackrel{q q_{1 \gamma}}{\longrightarrow} H^{Q-n}\left(E_{1}\left|A, E_{1}^{0}\right| A \cup E_{1} \mid B\right) \\
& \text { " } \phi_{2 *} \\
& H_{n-q_{2}}\left(X-B, X-A ; \mathcal{O}_{2}^{*}\right) \stackrel{\gamma_{X}}{\approx} H^{q+q_{2}-n}\left(A, B ; \mathcal{O}^{*}(\tau) \times \mathcal{O}_{2}^{*}\right),
\end{aligned}
$$

where $\phi_{1}^{*}, \phi_{2^{*}}$ are the relative Thom isomorphisms and $\gamma_{x}$ the AlexanderSpanier duality map for the manifold $X$.

The proof uses Theorem 2 and properties of the slant product.

(7) Corollary. Let $X$ be a compact manifold which is a complex and let $\mu_{1}, \mu_{2}$ be two microbundles over $X$ such that $\tau \oplus \mu_{1} \oplus \mu_{2}$ has trivial stable fibre homotopy type. Let $(A, B)$ be a pair of subcomplexes in $X$. Then $(A, B)$ is r-imbedded in $X$ with radial neighbourhoods $(U, V)$, say, and $T_{\mu_{1}}(A, B)$ and $T_{\mu_{2}}(X-V, X-U)$ are $S$-duals.

\section{REFERENCES}

1. J. W. Alexander, On the deformations of an n-cell, Proc. Mat. Acad. Sri. U.S.A. 9 (1923), 406-407.

2. M. F. Atiyah, Thom complexes, Proc. London Math. Soc. 11 (1961), 291-310.

3. M. W. Hirsch, On nonlinear cell bundles, (Unpublished).

4. P. Holm, Microbundles and bundles, Bull. Amer. Math. Soc. 72 (1966), 545-548.

University of California, Berkeley 